\title{
Hydrophobic Ice Confined between Graphene and
}

\section{$\mathrm{MoS}_{2}$}

\author{
Pantelis Bampoulis, ${ }^{* \dagger, \ddagger}$ Vincent J. Teernstra, ${ }^{\dagger}$ Detlef Lohse, ${ }^{\ddagger}$ Harold J. W. \\ Zandvliet, $^{\dagger}$ and Bene Poelsema ${ }^{\dagger}$ \\ $\dagger$ Physics of Interfaces and Nanomaterials, MESA + Institute for Nanotechnology, \\ University of Twente, P.O.Box 217, 7500AE Enschede, The Netherlands. \\ $\ddagger$ Physics of Fluids and J.M. Burgers Centre for Fluid Mechanics, MESA+ Institute for \\ Nanotechnology, University of Twente, P.O.Box 217, 7500AE Enschede, The Netherlands. \\ \Max Planck Institute for Dynamics and Self-Organization, Am Fassberg, 37077 \\ Göttingen, Germany \\ E-mail: p.bampoulis@utwente.nl
}


This article may be downloaded for personal use only. Any other use requires prior permission of the author and ACS publications. The following article appeared in . Phys. Chem. C, 2016, 120 (47), pp 2707927084 and may be found at http://pubs.acs.org/doi/abs/10.1021/acs.jpcc.6b09812.

\begin{abstract}
The structure and nature of water confined between hydrophobic molybdenum disulfide $\left(\mathrm{MoS}_{2}\right)$ and graphene $(\mathrm{Gr})$ are investigated at room temperature by means of atomic force microscopy (AFM). We find the formation of two dimensional (2D) crystalline ice layers. In contrast to the hexagonal ice 'bilayers' of bulk ice, these 2D crystalline ice phase consists of two planar hexagonal layers. Additional water condensation leads to either lateral expansion of the ice layers or to the formation of 3D water droplets on top or at the edges of the two-layer ice, indicating that water does not wet these planar ice films. The results presented here are in line with a recent theory suggesting that water confined between hydrophobic walls forms $2 \mathrm{D}$ crystalline two-layer ice with a non-tetrahedral geometry and intrahydrogen bonding. The lack of dangling bonds on either surface of the ice film gives rise to a hydrophobic character. The unusual geometry of these ice films is of great potential importance in biological systems with water in direct contact with hydrophobic surfaces.
\end{abstract}

\title{
Introduction
}

The properties and structure of water at planar surfaces have been extensively investigated using surface science techniques ${ }^{115}$ due to enormous interest in many fields including environmental sciences, lubrication, ${ }^{[6}$ nanofluidics ${ }^{7}$ and biology. ${ }^{8}$ The structure and nature of water is often affected by the surface's structure and wettability, pressure and temperature. For example, due to the strong binding of water to hydrophilic substrates, the structure of water near the surface is influenced by the substrate. This often results in non-tetrahedral 
bonding geometries, as has been observed for crystalline hydrophobic ice films grown on $\operatorname{Pt}(111)$ at cryogenic temperatures. $\underline{[}$

The lack of bonding of water to a hydrophobic substrate as well as stratification induced by the substrate (in its vicinity) can also lead to the growth of ice films with a non-tetrahedral geometry and remarkable properties. Kimmel et al.10 observed metastable crystalline ice grown at 100-135 K on $\mathrm{Gr} / \mathrm{Pt}(111)$. This crystalline ice consists of two flat hexagonal layers of water molecules. The hexagons of each layer are placed directly on top of each other. In this structure each molecule forms three in plane hydrogen bonds with each nearest neighbors and one out of plane hydrogen bond with the molecule in the opposite layer. This way the number of hydrogen bonds is maximized. There are no dangling bonds on either surface of the ice. The ice is considered hydrophobic since adding a third water layer is energetically not favorable. Water molecules that are located on top of the double ice layer will either diffuse towards the edge of the ice and incorporate into the double layer or will form 3D clusters. Similar ice films have also been observed on a $\mathrm{Au}(111)$ substrate ${ }^{11}$ at cryogenic temperatures and it has been hypothesized to exist on any hydrophobic planar surface due to the weak (van der Waals) interactions of the water molecules with the atoms of the surface.10 Molecular dynamics predicted the existence of these ice films in confined hydrophobic geometries ${ }^{12}$ that can be stable at room temperature, $\stackrel{1013}{13}$ but they have yet to be experimentally observed.

It is well known that the properties of confined water are in many cases dramatically different from their bulk counterpart. These properties include complex phase behavior, non-tetrahedral bonding geometry, novel phase transitions and anomalous self diffusion. $13+18$ Even though most knowledge comes from MD simulations and DFT calculations, recent experimental work of confined water in carbon nanotubes and Gr (slightly hydrophobic with bulk contact angle ranging from $70^{\circ}$ to $90^{\circ}$ ) (and other $2 \mathrm{D}$ materials) has provided information on the state of water confined between hydrophilic/hydrophobic $\frac{19}{24}$ and hydrophobic/hydrophobic $\frac{25 \mid 26}{}$ interfaces. For instance, Algara-Siller et al. ${ }^{26}$ provided evidence that water confined between two graphene surfaces acquires a square lattice, attributed to 
the large exerted pressure induced by the graphene surfaces. Despite significant efforts a systematic understanding of the influence of confinement on this rich behavior is still lacking. Understanding the vast amount of ice phases and the dynamics of ice structures is crucial for many fields including life sciences, environmental sciences, condensed matter physics, lubrication and nanofluidics. For example, ice layers on surfaces are used for the preparation of supported metal ${ }^{27}$ and oxide nanoparticles. $\frac{28}{}$ Moreover, water diffusion in hydrophobic nanopores is of great relevance for biological systems and nanofluidics. ${ }^{8}$ In the case of $2 \mathrm{D}$ materials, water adsorbates or intercalates can influence their electronic properties. For example, ice on Gr or under the Gr can electronically dope the Gr. $\stackrel{29 \mid 30}{ }$ The type of doping and level depends crucially on the structure and state of the ice.

In this paper we provide compelling experimental evidence that water confined at room temperature between $\mathrm{Gr} / \mathrm{MoS}_{2}$ forms a unique two-layer (2L) structure with low surface energy due to intrahydrogen bonding inside the ice. Our findings are in line with recent theoretical works, which have predicted the existence of a hydrophobic double layer ice with non-tetrahedral geometry in a hydrophobic confinement and at room temperature. $\frac{1013}{}$ Our work paves the way towards an understanding of the complex behavior of confined water structures at RT.

\section{Methods}

Exfoliated Gr flakes (cleaved from highly oriented pyrolytic graphite (HOPG ZYA grade, MikroMasch), consisting of one or a few layers are deposited on top of a freshly cleaved synthesized $2 \mathrm{H}-\mathrm{MoS}_{2}$ (2D Semiconductors) substrate. Like Gr, $\mathrm{MoS}_{2}$ is considered to be hydrophobic. The reported contact angles, for water droplets on this surface, are in the range of 70-90 $\stackrel{31}{31}$ Optical Microscopy (DM2500H materials microscope, Leica, Germany) was used to locate the Gr flakes. Optical Microscopy and tapping mode atomic force microscopy, AFM, (Agilent 5100 atomic force microscope, Agilent) was used to determine the exact 
number of Gr layers (see supporting information 1). AFM imaging on the Gr/MoS $\mathrm{M}_{2}$ system was performed using Nanosensors SSS-FMR-10 with a nominal spring-constant of $2.8 \mathrm{~N} / \mathrm{m}$ and resonance frequency of $75 \mathrm{kHz}$. Water droplets and layers on top of the $\mathrm{Gr}$ or the $\mathrm{MoS}_{2}$ substrates require softer cantilevers in order to be properly imaged without affecting their shape and size. ${ }^{32}$ In these experiments we have used MikroMasch HQ:NSC36/AL BS C cantilevers with a nominal spring-constant of $0.6 \mathrm{~N} / \mathrm{m}$ and a resonance frequency of 65 kHz. The AFM was operated in intermittent contact amplitude modulated mode. The cantilever was excited with a frequency slightly above its resonance frequency. When the tip is in the proximity of the surface, the oscillation amplitude is reduced as compared to the free amplitude, $A_{\text {Free }}$, owing to the force field induced by the sample. Scanning of the surface's topography is achieved by keeping a constant setpoint amplitude $\left(A_{S P}\right)$. For the case of droplets and water layers on the air/substrate interface (without the graphene cover) $A_{\text {Free }}$ was kept below $10 \mathrm{~nm}$ and $A_{S P}$ was set to $0.95 \times A_{\text {Free }}$. This way a potential capture of the tip from the interfacial water structures was avoided. Furthermore, AFM imaging was performed inside an environmental chamber. The relative humidity $(\mathrm{RH})$ inside the glove box and the AFM environmental chamber was controlled by an adjustable $N_{2}$ flow which either bubbled through a water bottle or directly purged into the chamber and was measured using a humidity sensor (SENSIRION EK-H4 SHTXX, Humidity Sensors, Eval Kit, SENSIRION, Switzerland), which has an accuracy of $1.8 \%$ between 10-90 \% RH. We emphasize that the experiments have been performed at RT.

\section{Results and Discussion}

Figure 1a describes the experimental process. $\mathrm{MoS}_{2}$ and thin Gr flakes were initially exposed to high (80-90\%) RH for a short period of time (5-10 mins) in a home built glove box. Water adsorbs on both surfaces, see the supporting information 2 for details. The Gr flakes are then deposited on top of the $\mathrm{MoS}_{2}$ substrate. The adsorbed water is confined between the 
two surfaces and the system is ready to be imaged by AFM, as can be seen in figure 1c (we note here that samples made at low (1-30\%) RH do not show any water structures at the interface, see figure 1b). In contrast to water structures on the free graphite or $\mathrm{MoS}_{2}$ substrates (see supp info 2), the confined water films are stable with well defined faceted edges, suggesting that they are ordered, i.e. crystalline. AFM phase images (see inset of figure 1c) suggest that these water structures are indeed between $\mathrm{Gr}$ and $\mathrm{MoS}_{2}$. In general, zero to little phase contrast $\left(\sim 0-1^{o}\right)$ is observed, which indicates that the probing tip always interacts with the same surface, i.e. Gr.

Occasionally, we observe that some of the water structures slowly grow over time, see figures $2 \mathrm{a}$ and $2 \mathrm{~b}$. We often observe that a propagating front -of a lower height contrast with respect to the initial structure- leads the growth, figure 2a. A movie of the growing ice structure is shown in the supporting information 3. The very rich growth and melting dynamics of these water structures will be discussed in detail in future work. Two height levels can also be seen at various other images and at different areas and samples, for instance, figure 2c shows islands under a few layers of Gr with two distinct height levels.

Cross-sections taken across the water films, figure $2 \mathrm{~d}$, reveal a total height difference with respect to their surroundings of $0.56 \mathrm{~nm} \pm 0.02 \mathrm{~nm}$ (black curve). The cross-sections also reveal that each level (layer) has a height of about $0.28 \mathrm{~nm}$ (red and blue curves). These values do not correspond at all to the height of the bilayer of hexagonal ice 'Ih-ice' $(0.37 \mathrm{~nm})$ or any multiples of it. However, they are rather close to the size of planar ice films, 0.285 nm. Furthermore, they clearly have a hexagonal symmetry as can be seen in the histogram of figure 3a, where a distribution of the angles between two adjacent island edges is shown. A predominant peak at around $120^{\circ}$ is clearly observed, suggesting a hexagonal symmetry. Furthermore, shoulders at approximately $90^{\circ}$ and $60^{\circ}$ are observed. Often, an elongation of the islands is observed, similar to what we saw for water condensation on the bare $\mathrm{MoS}_{2}$ surface, see supporting info 2 , indicating that the growth of the islands is kinetically limited. 

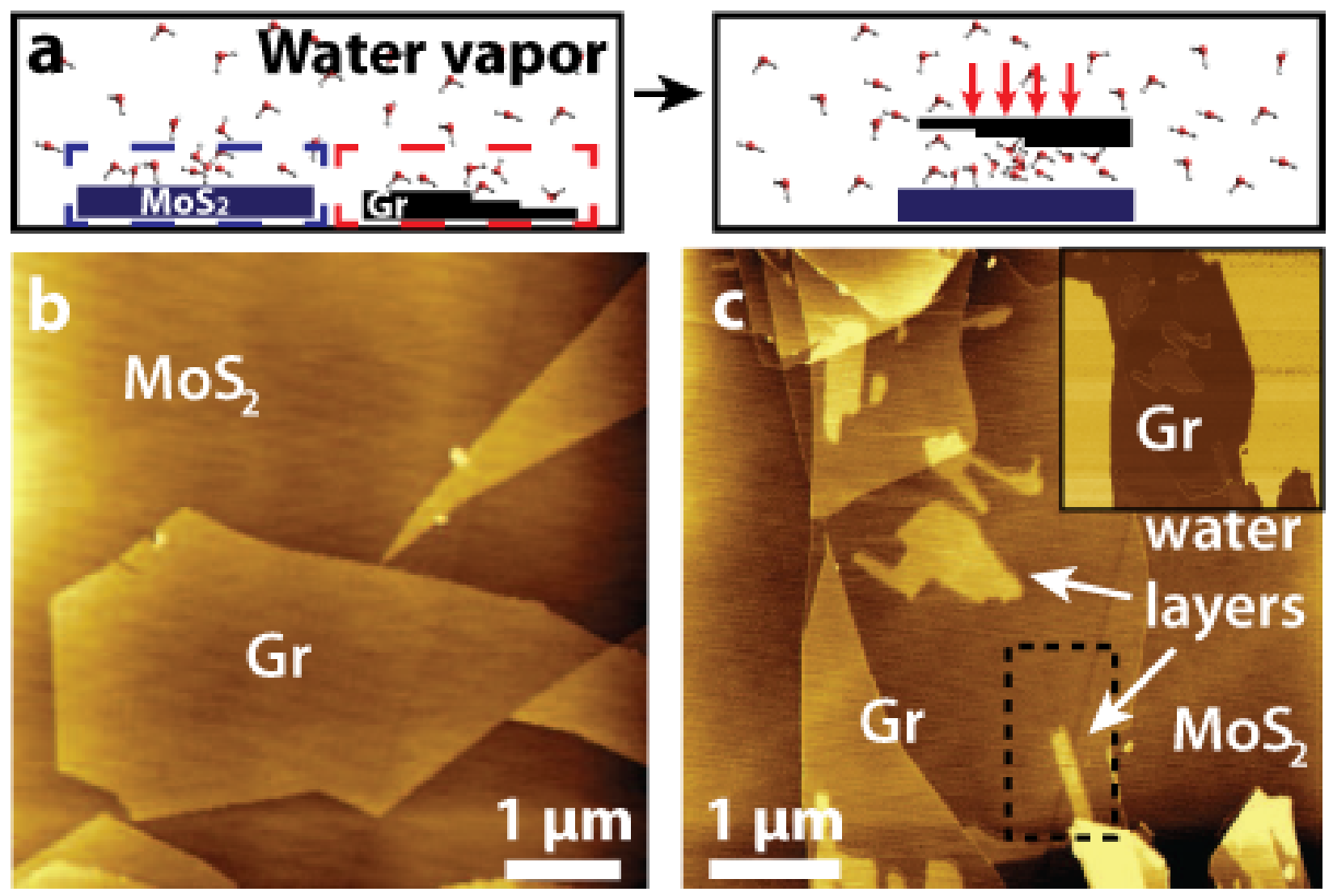

Figure 1: (a) Cartoon of the experimental procedure. The Gr and the $\mathrm{MoS}_{2}$ surfaces are exposed to a high RH environment (about 80\%) for a short amount of time (5-10 minutes). Water adsorbs on both surfaces leading to the formation of water layers and droplets. When the two surfaces are brought together these water structures are confined between the two materials. (b) Monolayer Gr on $\mathrm{MoS}_{2}$ deposited under 30\% RH. (c) Monolayer and few layers Gr on $\mathrm{MoS}_{2}$ deposited after the two surfaces were exposed for 5 minutes to high (80-90\%) $\mathrm{RH}$. Water layers are confined between the two surfaces. The inset is the corresponding phase image and it reveals little phase contrast between the water layers and the Gr surface but huge contrast between $\mathrm{Gr}$ and $\mathrm{MoS}_{2}$.

Our results clearly demonstrate the existence of planar two-layer (2L) crystalline ice films with a hexagonal symmetry in a hydrophobic confinement and at RT. The observed ice structures are reminiscent of a novel ice phase, which has been first observed at low temperature experiments on hydrophobic substrates, such as graphene.10111 As has been described first elsewhere $\frac{10112}{\sqrt{10}}$ these ice films consist of two hexagonal planar layers in registry with each other and with a non-tetrahedral geometry. Every water molecule of the $2 \mathrm{~L}$ ice forms four hydrogen bonds with its neighbors, three in the same plane and one out of plane 

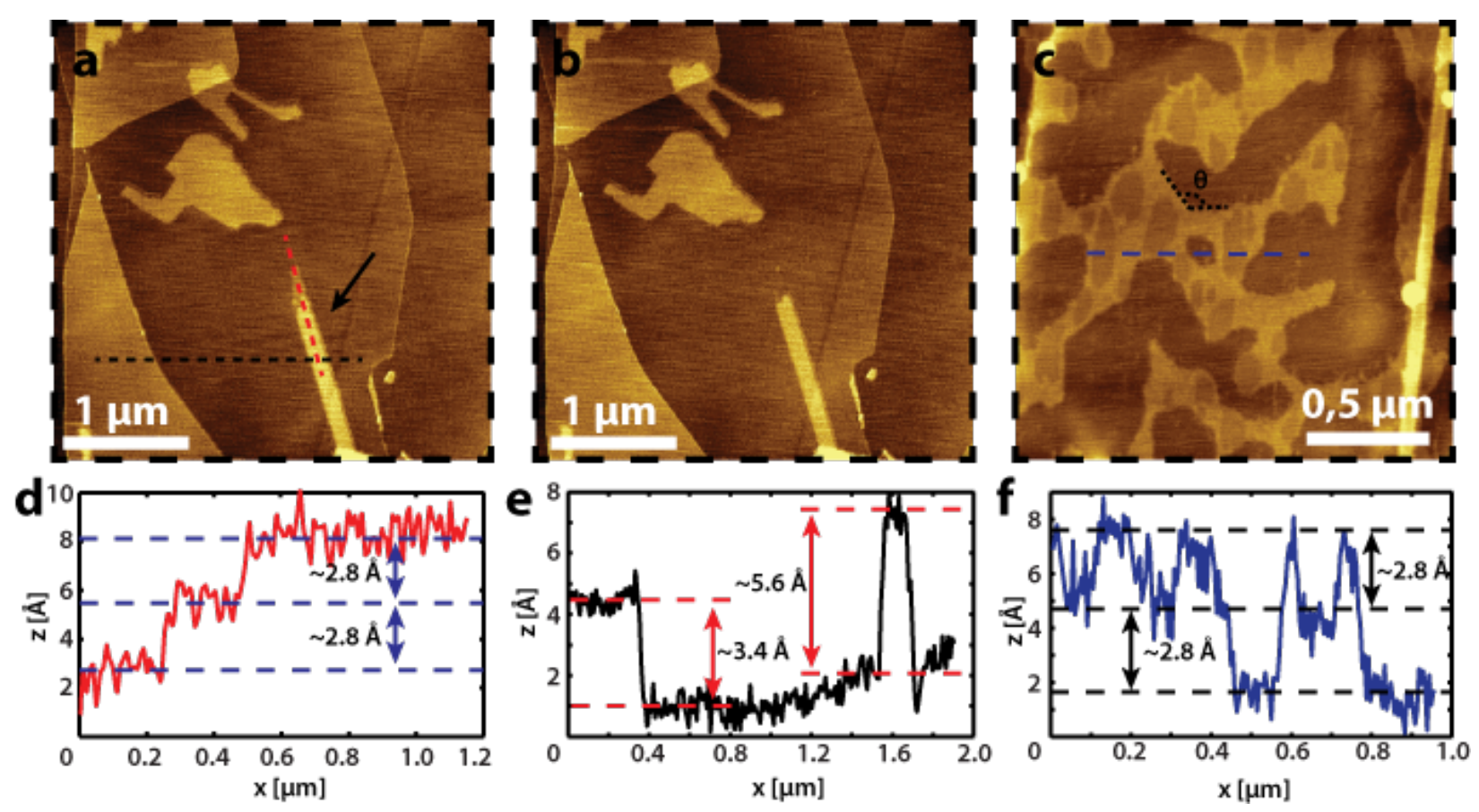

Figure 2: (a) and (b) are snapshots during the growth of a water layer indicated with the black arrow. (c) Water layers under few layers of Gr. (d) and (e) and (f) are line profiles taken across the structures of panel (a) (red and black, respectively) and panel (c) (blue) revealing that these structures consist of two layers of water.

with the water molecule of the opposite layer, leading to a self-closed hydrogen bonding network with low surface energy due to the maximized number of hydrogen bonds. ${ }^{10}$ The lattice constant of the $2 \mathrm{~L}$ ice is $0.48 \mathrm{~nm}, \frac{10}{10}$ in contrast to $0.45 \mathrm{~nm}$ of the bilayer $\mathrm{Ih}$. A cartoon of the top and the side view of the 2L ice structure are shown in figure $3 \mathrm{~b}$.

The total area covered by water depends on the exposure time and the distance of the sample from the inlet of the water vapor into the glove box. Longer exposure and at a closer proximity to the inlet results in a larger lateral expansion of the two dimensional ice layers and often to a higher density of water droplets, see figure 4a. Figure $4 \mathrm{~b}$ shows that all the 2L ice structures and the water droplets are under the Gr, no phase contrast is observed. Water droplets on top of the Gr show strong phase contrast $\left(\sim 20^{\circ}\right)$ and can be dragged by the AFM tip. We have never observed the growth of a third ice layer, which indicates that two layer thick ice is energetically more favorable than three layers thick ice. Occasionally 3D droplets of various sizes are observed on top or at the edges of the $2 \mathrm{~L}$ 

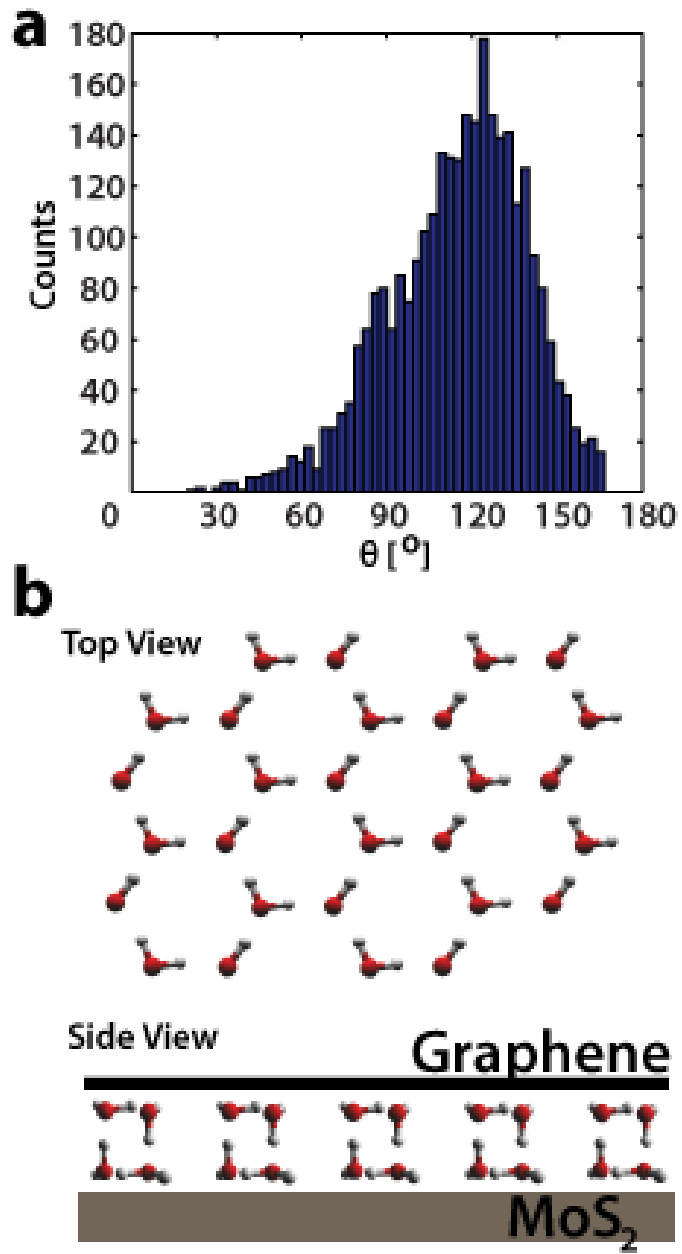

Figure 3: (a) Histogram of the distribution of the angles measured between two adjacent edges of the water layers, as indicated in figure 2c. The histogram shows a predominant peak at $120^{\circ}$. (b) Top and side view of the structural model of the $2 \mathrm{~L}$ ice. The red and white spheres represent $\mathrm{O}$-atoms and $\mathrm{H}$-atoms, respectively.

ice, figure 4. DFT calculations ${ }^{11}$ demonstrate that $1 \mathrm{~L}$ or $3 \mathrm{~L}$ thick ice are energetically less favored when compared to 2L thick ice: $3 \mathrm{~L}$ ice is less stable than $2 \mathrm{~L}$ ice by not less than 0.1 $\mathrm{eV} / \mathrm{H}_{2} \mathrm{O}, 11$ although it is slightly more stable than $1 \mathrm{~L}$ ice. This last finding contradicts our observations since we have never observed any $3 \mathrm{~L}$ ice structures, while in contrast, we have seen in many occasions $1 \mathrm{~L}$ ice structures. We attribute this difference to the fine details of the hydrophobic confinement. The DFT calculations have been performed for ice structures on a hydrophobic solid-vacuum interface, however in our case the second hydrophobic wall might induce another limitation making the 3 layer ice energetically less unfavorable than 

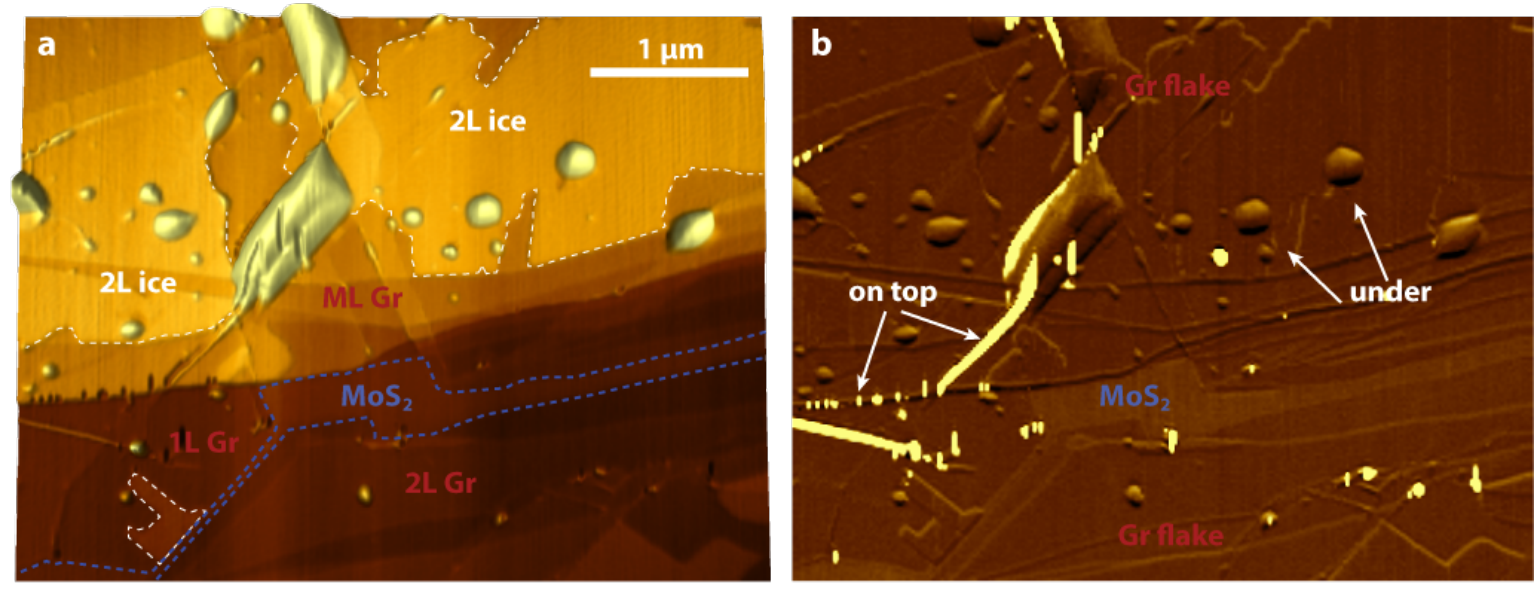

Figure 4: (a) A large scale AFM image of a Gr flake with various thickness on $\mathrm{MoS}_{2}$. $2 \mathrm{~L}$ ice marked with the white dashed lines are under the Gr flake. Occasionally various sizes of water droplets are on top and at the edges of the $2 \mathrm{~L}$ ice. Droplets between $\mathrm{Gr}$ and $\mathrm{MoS}_{2}$ are also observed. (b) The corresponding phase image, both the 2L ice and droplets reveal no apparent phase contrast with respect to the Gr surface. For comparison, water droplets on the $\mathrm{MoS}_{2}$ and Gr reveal a strong phase contrast.

the $1 \mathrm{~L}$ ice. The surface free energy of the $2 \mathrm{~L}$ ice is low. The $2 \mathrm{~L}$ ice films are only weakly interacting with the substrate or the Gr cover. Owing to these weak interactions, small $2 \mathrm{~L}$ ice islands are very mobile, see supporting information 3.

The lack of $\mathrm{OH}$ dangling bonds on either of the two surfaces of the ice, gives the 2L ice a hydrophobic character. Water molecules that arrive on top of the two layer ice are weakly bound and will prefer either to diffuse towards the edges where they become part of the ice structure or form a 3D non wetting structure. $\frac{910}{10}$ This is also consistent with our system, since there is no observation of a layer by layer growth but rather a lateral expansion and/or the observation of $3 \mathrm{D}$ droplets on top or at the edges of the $2 \mathrm{~L}$ ice. To test whether the droplets are indeed on top of the ice layers and not surrounded by ice, we have measured the droplet height as a function of footprint diameter and compared it to sessile water droplets on the $\mathrm{MoS}_{2}$ surface, figures $5 \mathrm{a}$ and $\mathrm{b}$. Note here that large droplets under the graphene display a polygonal shape, induced by the graphene cover. In order to avoid any misinterpretation of our results we have restricted our analysis to droplets with a spherical shape, see figure 5a. A clear difference is observed in figures $5 \mathrm{a}, 5 \mathrm{~b}$ and $5 \mathrm{c}$, suggesting that the droplets 

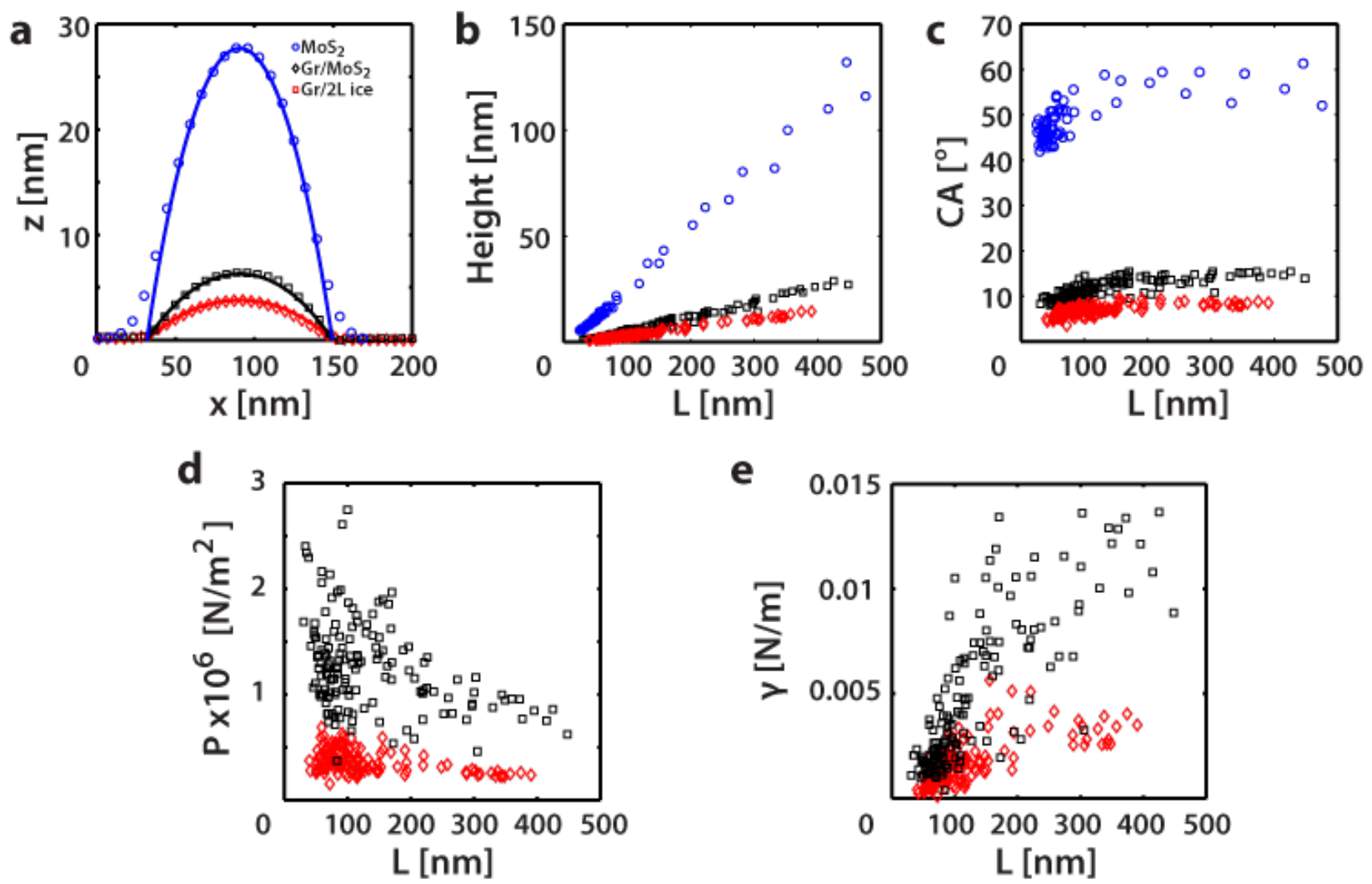

Figure 5: (a) Cross sections of droplets between $\mathrm{Gr} / \mathrm{MoS}_{2}$ (black), Gr/2L ice (red) and on the bare $\mathrm{MoS}_{2}$ surface (blue). The solid lines are spherical cap fits. (b) Height (H) vs. footprint diameter (L) and (c) contact angle (CA) vs. footprint diameter (L) for all three cases. (d) Pressure (P) vs. footprint diameter (L) and (e) Adhesion energy ( $\gamma$ ) vs. footprint diameter (L) for droplets between $\mathrm{Gr} / \mathrm{MoS}_{2}$ (black) and $\mathrm{Gr} / 2 \mathrm{~L}$ ice (red).

are in between different surfaces, i.e. Gr/MoS 2 (black points in figure 5) and $\mathrm{Gr} / 2 \mathrm{~L}$ ice (red points in figure 5). It is also evident from figure $5 \mathrm{c}$ that the contact angle of a water droplet between $\mathrm{Gr}$ and $\mathrm{MoS}_{2}$ (black points) is substantially lower than the contact angle of large sessile water droplets on $\mathrm{MoS}_{2}$ (blue points). ${ }^{31}$ In order to check this in more detail we have measured water droplets on the bare $\mathrm{MoS}_{2}$ surface and compared their sizes and contact angles with the confined droplets. We measured contact angles in the range of $60^{\circ}$, i.e., very close but slightly smaller than the reported values for large sessile water droplets. Note here that convolution effects have been taken into account according to ref. ${ }^{33}$ We note that especially on the free sessile droplets, different tip-surface interactions can overestimate or underestimate up to some extent these measurements. The large difference in contact 
angles between sessile droplets on the $\mathrm{MoS}_{2}$ surface and droplets confined between $\mathrm{MoS}_{2}$ and $\mathrm{Gr}$ is attributed to confinement effects. The pressure induced by the confinement is substantial ${ }^{26 / 34}$ and therefore capable of deforming small droplets, resulting in the reduction of their contact angle.

The diameter and height of droplets confined between a 2D membrane, such as Gr, and a supporting substrate depend on the number of molecules, the adhesion energy between the membrane and the substrate, in that case either $\mathrm{MoS}_{2}$ or $2 \mathrm{~L}$ ice, and the elastic properties of the membrane. The ratio of the height vs the diameter is determined by the balance between van der Waals interactions and the elastic energy of graphene. ${ }^{34}$ It has been proposed

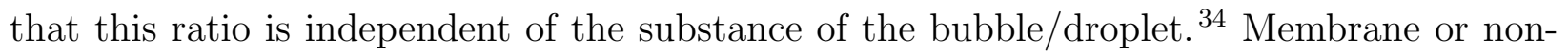
linear plate theories are typically used to determine the adhesion energy of Gr with its substrate, by the use of a trapped bubble or blister. $\frac{35136}{}$ Yue et al. ${ }^{35}$ demonstrated that for monolayer Gr covering relative big bubbles the membrane analysis is sufficient. This analysis uses a spherical cap approximation but does not take into account the small but finite bending stiffness of Gr. Therefore it does not describe the bubble's edges accurately but nevertheless provides a simple solution. Using membrane analysis the pressure inside the bubble is obtained by, 35

$$
P=\frac{E_{2 D} H^{3}}{\Phi L^{4}}+P_{0}
$$

where $E_{2 D}$ is the 2D Young's modulus of single layer $\mathrm{Gr}, \stackrel{37}{\mathrm{Bn}}$ and $\mathrm{L}$ the height and footprint diameter of the droplet, $P_{0}$ is the external pressure, $\Phi=\frac{75\left(1-\nu^{2}\right)}{8\left(23+18 \nu-3 \nu^{2}\right)}$ and $\nu$ is Poisson's ratio equal to 0.16 for monolayer Gr. We have estimated the pressure inside the droplets between monolayer Gr and $\mathrm{MoS}_{2}$ and monolayer Gr and 2L ice using equation (1), see figure $5 \mathrm{~d}$. In both cases smaller droplets display larger pressures, the pressure decays fast as the size increases and saturates at approximately $1 \times 10^{6} \mathrm{~N} / \mathrm{m}^{2}$ for $\mathrm{Gr} / \mathrm{droplet} / \mathrm{MoS}_{2}$ and $3 \times 10^{5}$ $N / m^{2}$ for $\mathrm{Gr} /$ droplet/2L ice.

Using the ideal gas law, the Gr-substrate adhesion energy is directly related to the equilibrium droplet size, since it is a result of the potential energy of the droplet balanced by 
the interfacial energy. The adhesion energy $(\gamma)$ is derived ${ }^{35}$ to be equal to:

$$
\gamma=\frac{5 E_{2 D} H^{4}}{8 \Phi L^{4}}
$$

The Gr substrate adhesion energy is shown in figure 5e for both cases as calculated from equation (2). It can be seen that $\mathrm{Gr}$ adheres better on the $\mathrm{MoS}_{2}$ substrate with an adhesion energy in the order of $\sim 0.01 \mathrm{~N} / \mathrm{m}$ whereas the $\mathrm{Gr} / 2 \mathrm{~L}$ ice adhesion energy is in the range of $\sim 0.003 \mathrm{~N} / \mathrm{m}$

\section{Conclusions}

Our scanning probe investigation of confined water is consistent with the existence of a nontetrahedral ice phase at RT in a hydrophobic confinement. This ice phase consists of two planar hexagonal ice layers. The water molecules of each layer of the ice are stacked on top of each other and have a self closed hydrogen bonding. This leads to weak interactions with the substrate and with other water molecules on top of the 2L ice. Our results are in line with recent MD simulations of ice films confined between hydrophobic walls and low temperature experiments of ice films on graphene and $\mathrm{Au}(111)$ substrates. This unusual geometry of the 2L ice may be of importance in biological samples, where water is in direct contact with hydrophobic surfaces, and may be also relevant for water structures on hydrophobic surfaces and at ambient conditions.

\section{Acknowledgements}

We would like to thank the Nederlandse Organisatie voor Wetenschappelijk Onderzoek (NWO, STW 11431) for financial support. 


\section{References}

(1) Hodgson, A.; Haq, S. Water adsorption and the wetting of metal surfaces. Surf. Sci. Rep. 2009, 64, 381-451.

(2) Thiel, P. A.; Madey, T. E. The interaction of water with solid surfaces: Fundamental aspects. Surf. Sci. Rep. 1987, 7, 211-385.

(3) Verdaguer, A.; Sacha, G. M.; Bluhm, H.; Salmeron, M. Molecular structure of water at interfaces: Wetting at the nanometer scale. Chem. Rev. 2006, 106, 1478-1510.

(4) Feibelman, P. J. The first wetting layer on a solid. Phys. Today 2010, 63, 34-39.

(5) Berkelaar, R.; Bampoulis, P.; Dietrich, E.; Jansen, H.; Zhang, X.; Kooij, E.; Lohse, D.; Zandvliet, H. Water-induced blister formation in a thin film polymer. Langmuir 2015, 31, 1017-1025.

(6) Lee, C.; Li, Q.; Kalb, W.; Liu, X. Z.; Berger, H.; Carpick, R. W.; Hone, J. Frictional characteristics of atomically thin sheets. Science 2010, 328, 76-80.

(7) Prakash, S.; Piruska, A.; Gatimu, E. N.; Bohn, P. W.; Sweedler, J. V.; Shannon, M. A. Nanofluidics: Systems and applications. IEEE Sens. J. 2008, 8, 441-450.

(8) Chaban, V. V.; Prezhdo, O. V. Water boiling inside carbon nanotubes: Toward efficient drug release. ACS Nano 2011, 5, 5647-5655.

(9) Kimmel, G. A.; Petrik, N. G.; Dohnlek, Z.; Kay, B. D. Crystalline ice growth on Pt(111): Observation of a hydrophobic water monolayer. Phys. Rev. Lett. 2005, 95.

(10) Kimmel, G. A.; Matthiesen, J.; Baer, M.; Mundy, C. J.; Petrik, N. G.; Smith, R. S.; Dohnlek, Z.; Kay, B. D. No confinement needed: Observation of a metastable hydrophobic wetting two-layer ice on graphene. J. Am. Chem. Soc. 2009, 131, 12838-12844. 
(11) Stacchiola, D.; Park, J. B.; Liu, P.; Ma, S.; Yang, F.; Starr, D. E.; Muller, E.; Sutter, P.; Hrbek, J. Water nucleation on gold: Existence of a unique double bilayer. J. Phys. Chem. C 2009, 113, 15102-15105.

(12) Koga, K.; Zeng, X. C.; Tanaka, H. Freezing of confined water: A bilayer ice phase in hydrophobic nanopores. Phys. Rev. Lett. 1997, 79, 5262-5265.

(13) Giovambattista, N.; Rossky, P. J.; Debenedetti, P. G. Phase transitions induced by nanoconfinement in liquid water. Phys. Rev. Lett. 2009, 102.

(14) Zhao, W. H.; Wang, L.; Bai, J.; Yuan, L. F.; Yang, J.; Zeng, X. C. Highly confined water: Two-dimensional ice, amorphous ice, and clathrate hydrates. Acc. Chem. Res. 2014, 47, 2505-2513.

(15) Cambré, S.; Schoeters, B.; Luyckx, S.; Goovaerts, E.; Wenseleers, W. Experimental observation of single-file water filling of thin single-wall carbon nanotubes down to chiral index (5,3). Phys. Rev. Lett. 2010, 104.

(16) Mukherjee, B.; Maiti, P. K.; Dasgupta, C.; Sood, A. K. Strong correlations and Fickian water diffusion in narrow carbon nanotubes. J. Chem. Phys. 2007, 126.

(17) Koga, K.; Tanaka, H.; Zeng, X. C. First-order transition in confined water between high-density liquid and low-density amorphous phases. Nature 2000, 408, 564-567.

(18) Cicero, G.; Grossman, J. C.; Schwegler, E.; Gygi, F.; Galli, G. Water confined in nanotubes and between graphene sheets: A first principle study. J. Am. Chem. Soc. 2008, 130, 1871-1878.

(19) Bampoulis, P.; Siekman, M. H.; Kooij, E. S.; Lohse, D.; Zandvliet, H. J. W.; Poelsema, B. Latent heat induced rotation limited aggregation in 2D ice nanocrystals. J. Chem. Phys. 2015, 143, 034702. 
(20) Severin, N.; Lange, P.; Sokolov, I. M.; Rabe, J. P. Reversible dewetting of a molecularly thin fluid water film in a soft graphene-mica slit pore. Nano Lett. 2012, 12, 774-779.

(21) Bampoulis, P.; Lohse, D.; Zandvliet, H. J. W.; Poelsema, B. Coarsening dynamics of ice fractals intercalated between graphene and mica. Appl. Phys. Lett. 2016, 108, 011601.

(22) Xu, K.; Cao, P.; Heath, J. R. Graphene visualizes the first water adlayers on mica at ambient conditions. Science 2010, 329, 1188-1191.

(23) Bampoulis, P.; Witteveen, J. P.; Kooij, E. S.; Lohse, D.; Poelsema, B.; Zandvliet, H. J. W. Structure and Dynamics of Confined Alcohol-Water Mixtures. ACS Nano 2016, 10, $6762-6768$.

(24) Dollekamp, E.; Bampoulis, P.; Poelsema, B.; Zandvliet, H.; Kooij, E. Electrochemically Induced Nanobubbles between Graphene and Mica. Langmuir 2016, 32, 6582-6590.

(25) Cao, P.; Xu, K.; Varghese, J. O.; Heath, J. R. The microscopic structure of adsorbed water on hydrophobic surfaces under ambient conditions. Nano Lett. 2011, 11, 55815586.

(26) Algara-Siller, G.; Lehtinen, O.; Wang, F. C.; Nair, R. R.; Kaiser, U.; Wu, H. A.; Geim, A. K.; Grigorieva, I. V. Square ice in graphene nanocapillaries. Nature 2015, $519,443-445$.

(27) Palmer, J. S.; Sivaramakrishnan, S.; Waggoner, P. S.; Weaver, J. H. Particle aggregation on dewetting solid water films. Surf. Sci. 2008, 602, 2278-2283.

(28) Kaya, S.; Sun, Y. N.; Weissenrieder, J.; Stacchiola, D.; Shaikhutdinov, S.; Freund, H. J. Ice-assisted preparation of silica-supported vanadium oxide particles. J. Phys. Chem. C 2007, 111, 5337-5344.

(29) Goncher, S. J.; Zhao, L.; Pasupathy, A. N.; Flynn, G. Substrate level control of the local doping in graphene. Nano Lett. 2013, 13, 1386-1392. 
(30) Bollmann, T. R. J.; Antipina, L. Y.; Temmen, M.; Reichling, M.; Sorokin, P. B. Holedoping of mechanically exfoliated graphene by confined hydration layers. Nano Res. 2015, 8, 3020-3026.

(31) Gaur, A.; Sahoo, S.; Ahmadi, M.; Dash, S.; Guinel, M.-F.; Katiyar, R. Surface energy engineering for tunable wettability through controlled synthesis of MoS2. Nano Lett. 2014, 14, 4314-4321.

(32) Lohse, D.; Zhang, X. Surface nanobubbles and nanodroplets. Rev. Mod. Phys. 2015, 87.

(33) Canet-Ferrer, J.; Coronado, E.; Forment-Aliaga, A.; Pinilla-Cienfuegos, E. Correction of the tip convolution effects in the imaging of nanostructures studied through scanning force microscopy. Nanotechnology 2014, 25.

(34) Khestanova, E.; Guinea, F.; Fumagalli, L.; Geim, A.; Grigorieva, I. Universal shape and pressure inside bubbles appearing in van der Waals heterostructures. Nat. Commun. 2016, 7 .

(35) Yue, K.; Gao, W.; Huang, R.; Liechti, K. M. Analytical methods for the mechanics of graphene bubbles. J. Appl. Phys. 2012, 112.

(36) Temmen, M.; Ochedowski, O.; Schleberger, M.; Reichling, M.; Bollmann, T. R. J. Hydration layers trapped between graphene and a hydrophilic substrate. New J. Phys. 2014,16 .

(37) Lee, C.; Wei, X.; Kysar, J. W.; Hone, J. Measurement of the elastic properties and intrinsic strength of monolayer graphene. Science 2008, 321, 385-388. 


\section{Supporting Information}

\section{Determination of the Graphene Flake Thickness}
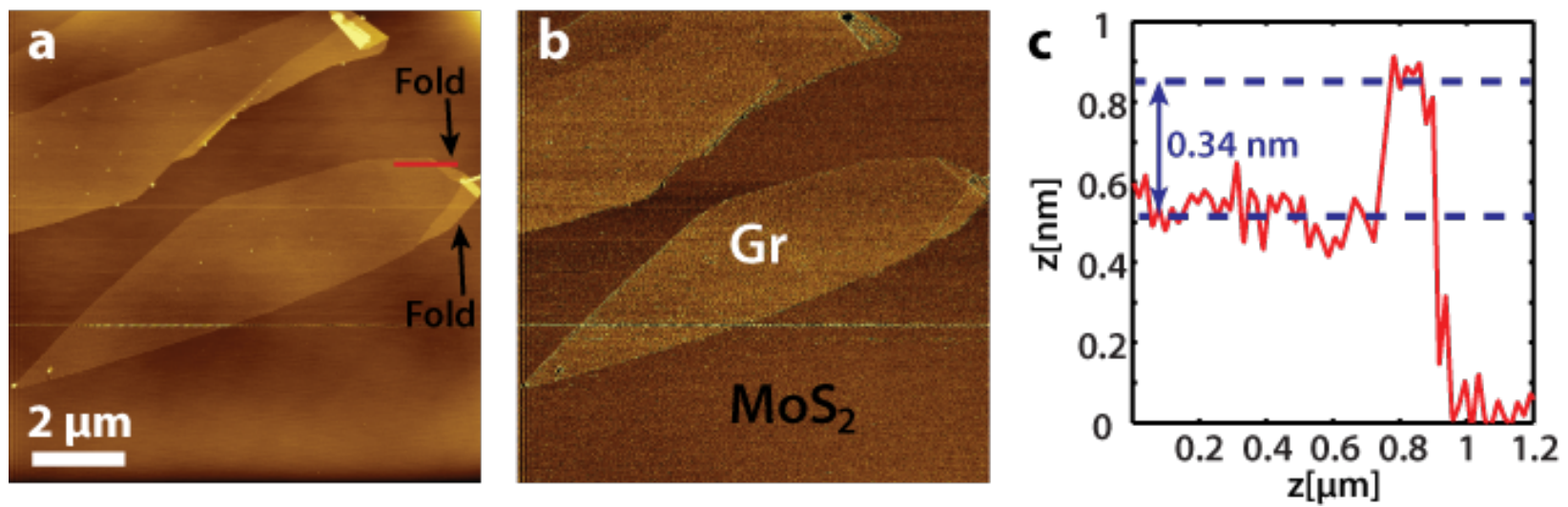

Figure 6: (a) Graphene flakes on the $\mathrm{MoS}_{2}$ surface. The graphene flake is folded at one of each sides. (b) The corresponding phase image of (a) revealing two distinct contrasts that correspond to the graphene and $\mathrm{MoS}_{2}$ regions. (c) Line profile of the graphene fold noted with the red solid line in panel (a).

In order to determine the thickness of the graphene flake on $\mathrm{MoS}_{2}$ we use AFM images. In principle, AFM cannot provide accurate height interpretation between two different materials, i.e. Gr and $\mathrm{MoS}_{2}$, due to the different tip-surface interactions between the two surfaces. In order to overcome this problem, we use regions where one of the sides of the graphene flake is folded, see for example figure 6 (a). These folds can be often found on exfoliated samples and are a product of the exfoliation process. They can be also manually created by scanning the edge of the graphene flake in contact mode. A line profile measurement across the fold, figure 6 (c), can then be used to exactly determine the thickness of the graphene flake, in this case the flake is a monolayer of graphene and thus the fold a bilayer.

\section{Adsorption of Water on Graphene and $\mathrm{MoS}_{2}$}

In order to clarify that water adsorbs on both HOPG (or Graphene flakes) and $\mathrm{MoS}_{2}$ surfaces we have exposed them at high relative humidity (80-90\%) for a couple of minutes and 

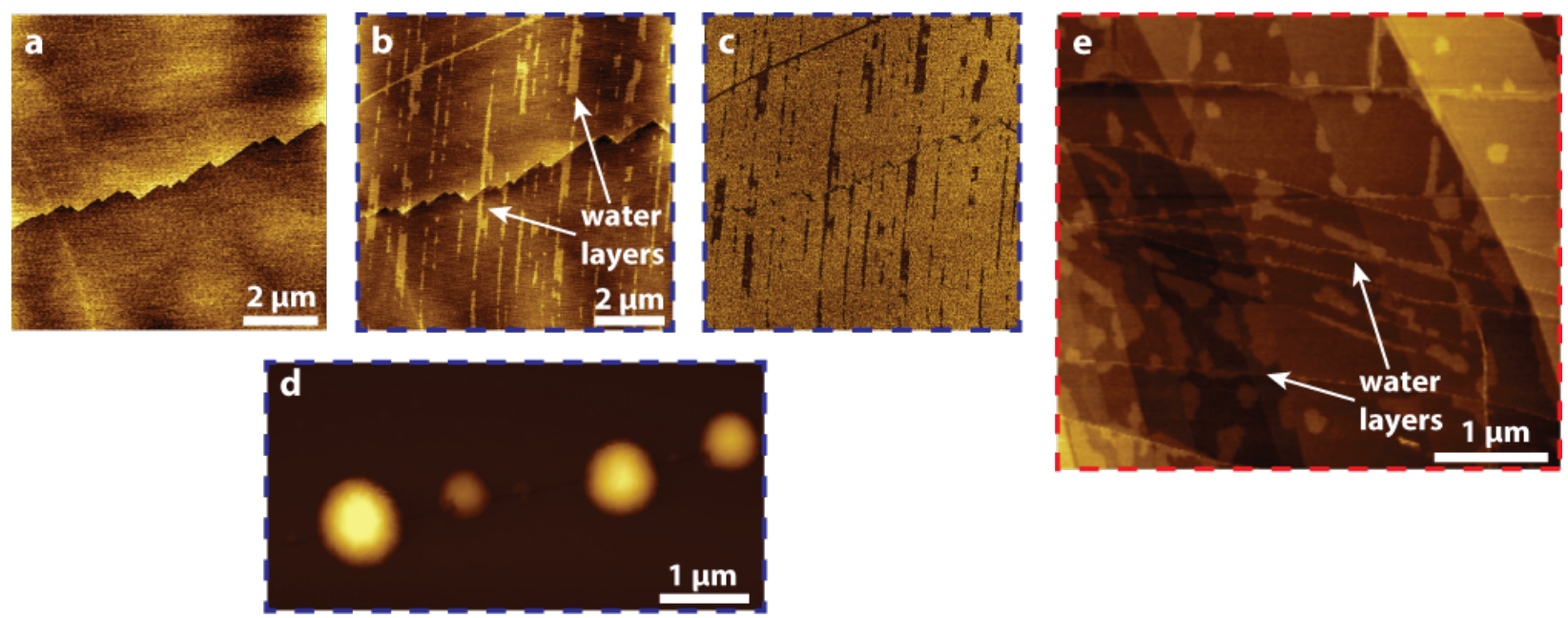

Figure 7: (a) AFM topographic image of a freshly cleaved $\mathrm{MoS}_{2}$ measured at ambient conditions. (b) The same location after exposing the surface to $85 \% \mathrm{RH}$ for 5 minutes. Elongated water layers are formed along the surface. (c) The corresponding phase image of (b), revealing sharp phase contrast between the water layers and the $\mathrm{MoS}_{2}$ surface. (d) Water droplets formed on the $\mathrm{MoS}_{2}$ surface after long exposure to high RH. (e) AFM image of an HOPG sample after exposure to water vapor for a couple of minutes. Water layers are visible on the whole surface.

subsequently imaged the surfaces with AFM. The results are shown in figure 7 . Water adsorbs in the form of layers or droplets at both surfaces depending on the exposure time but also at the surface's morphology. We would like to note here that in contrast to the well defined ice structures that are confined between $\mathrm{Gr}$ and $\mathrm{MoS}_{2}$, these water layers are very dynamic with lousy edges and can be easily disturbed and modified by the probing tip.

Note also that they exhibit a large phase contrast with respect to their surroundings and in contrast to the confined structures.

\section{Growth of Ice Crystals and Mobility of Small Clusters}

We have recorded the dynamics of the $2 \mathrm{~L}$ ice crystals by scanning continuously over a long period of time (3 days) with AFM. In figure 8 snapshots of the growth of a $2 \mathrm{~L}$ ice crystal is shown. The 2L ice crystals grows in one direction (different from the scanning direction by about $30^{\circ}$ ) acquiring an elongated form. This crystallite appears to grow at the expense of the 

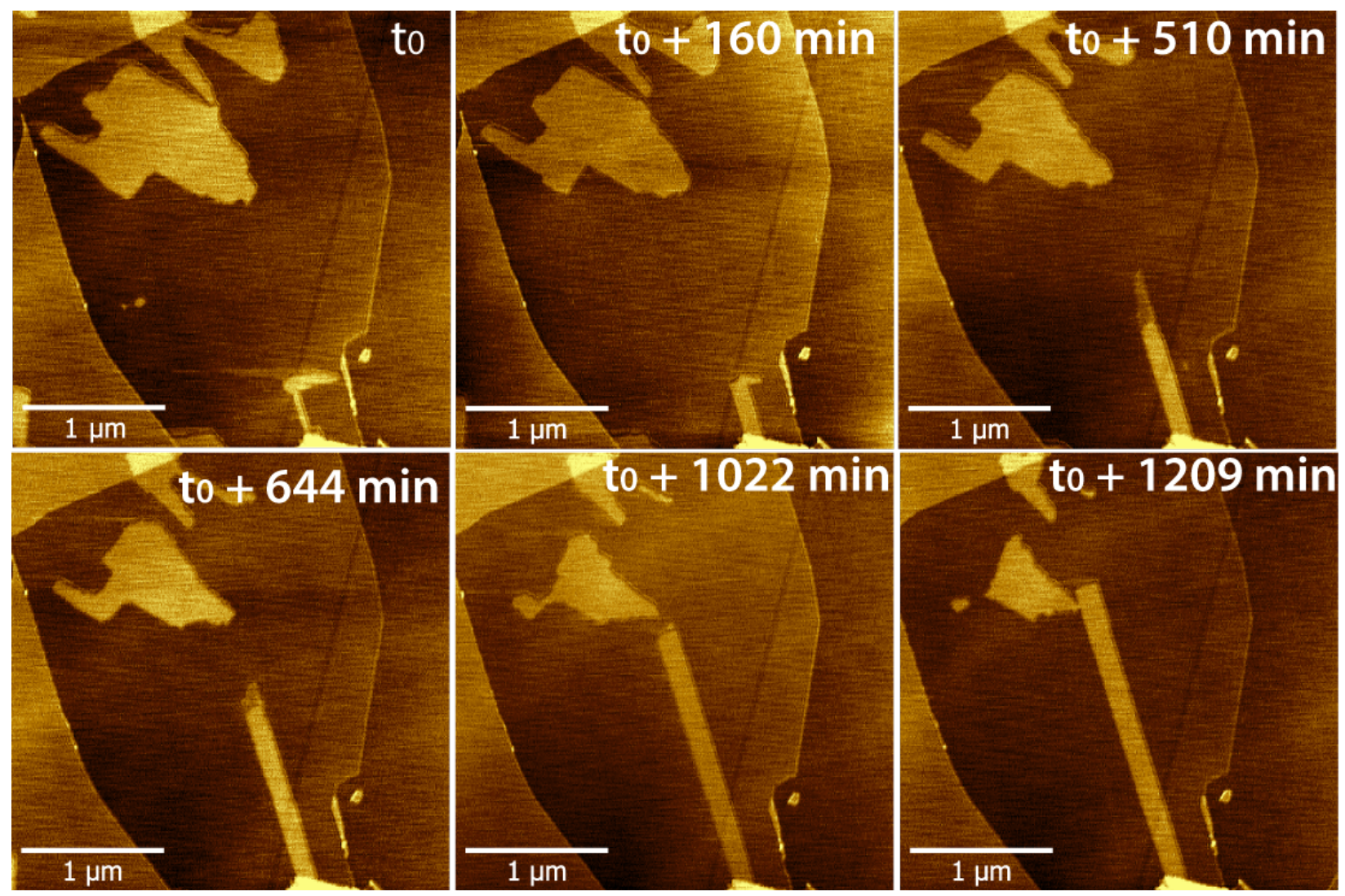

Figure 8: (a) AFM image snapshots of the growth of a 2L ice crystal. The 2L ice crystal grows at the expense of surrounding $2 \mathrm{~L}$ ice crystals.

surrounding crystals, indicating mass transport. As the crystal grows, often, a propagating 1 layer thick front is observed which acts as an apparent precursor of the $2 \mathrm{~L}$ ice. The complete movie is given as a suppl. movie. Similar dynamics have been observed in several samples. A detailed study of the growth and melting dynamics of the $2 \mathrm{~L}$ ice crystals will be part of a future work.

We have also recorded the mobility of small 2L ice crystals. Snapshots are given in figure 9. The $2 \mathrm{~L}$ ice crystals moves with steps up to 100 s of nanometer within a few minutes of measuring time. The high mobility of small clusters is explained by the weak interaction of the 2L ice with both surfaces $\left(\mathrm{MoS}_{2}\right.$ and $\left.\mathrm{Gr}\right)$. 


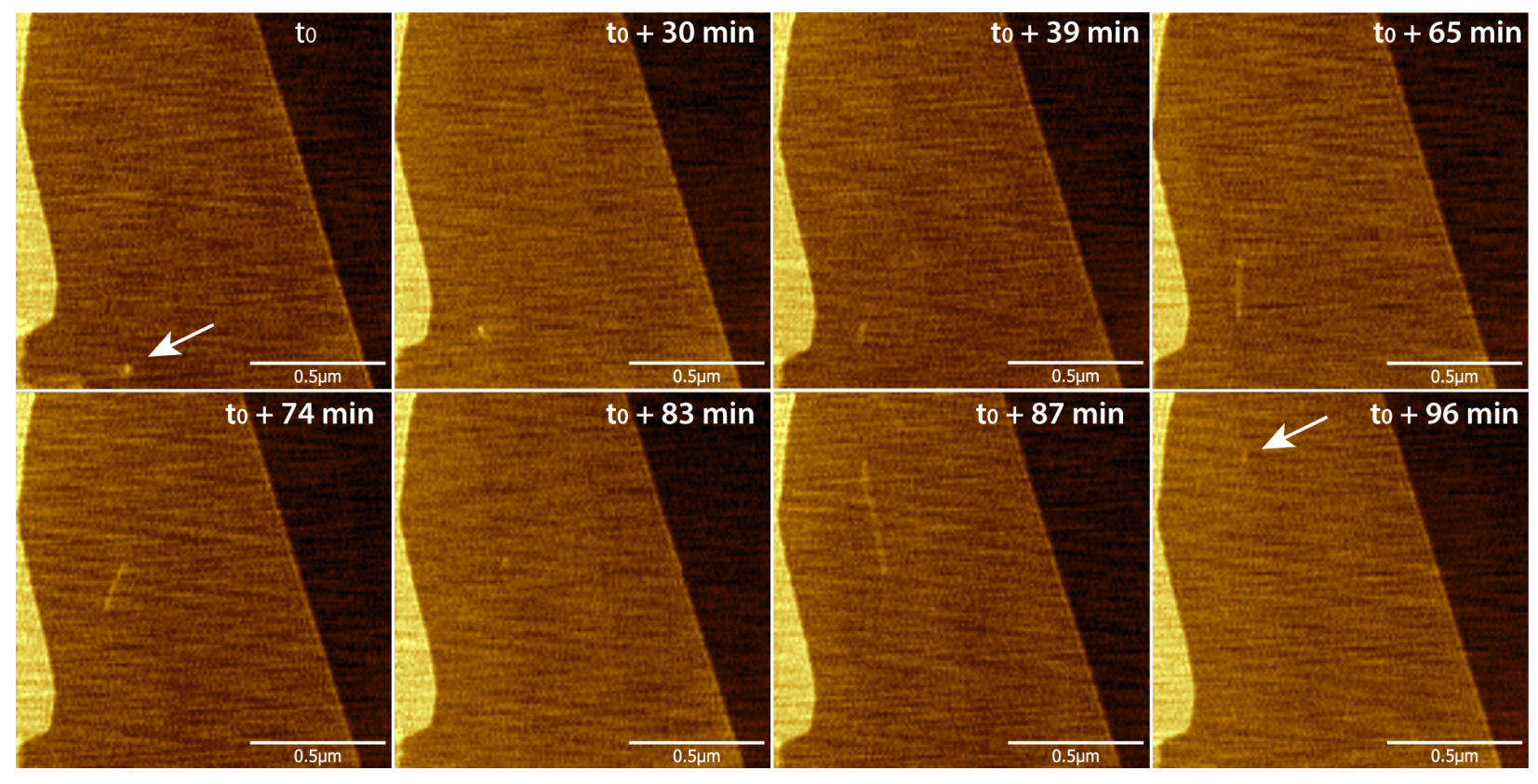

Figure 9: (a) AFM image sequence of a small 2L ice crystal. The ice crystal shows rapid movement in between the two surfaces. Eventually it escapes from the interface at the Gr $\mathrm{MoS}_{2}$ boundary. 